\title{
Treatment of presumed trematode-induced granulomatous anterior uveitis among children in rural areas of Egypt
}

\author{
Amgad El Nokrashy ${ }^{1,2,3} \cdot$ Waleed Abou Samra ${ }^{1}$ Doaa Sobeih ${ }^{4,5} \cdot$ Ali Lamin $\mathbb{1}^{2,3} \cdot$ Aya Hashish $^{1} \cdot$ Sahar Tarshouby ${ }^{1}$. \\ Susan Lightman ${ }^{2,3} \cdot$ Ashraf Sewelam $^{1}$
}

Received: 10 May 2018 / Revised: 26 December 2018 / Accepted: 24 February 2019 / Published online: 3 April 2019

(c) The Royal College of Ophthalmologists 2019

\begin{abstract}
Purpose The purpose of this study is to evaluate the efficacy of systemic antiparasitic medications alone or in combination with surgical aspiration in management of presumed trematode-induced anterior uveitis in children.

Patients and methods Prospective case series. Children who presented with anterior chamber (AC) granuloma were included in the study. All patients received antiparasitic treatment and after 2 weeks; patients were divided based on their clinical improvement in terms of the baseline granuloma area into two groups: group A $\left(<2.5 \mathrm{~mm}^{2}\right)$ who continued on antiparasitic medications only $(n=15)$ and group B $\left(\geq 2.5 \mathrm{~mm}^{2}\right)$ who underwent surgical aspiration $(n=15)$. Basic demographics data, visual acuity (VA), corneal thickness, granuloma area and AC activity (cells and flare) were recorded and analysed. Systemic work-up including stool and urine analysis, full blood count, chest X-ray and schistosomiasis titre were performed.

Results Thirty eyes of 30 patients were included in the study with a mean age of $13.4 \pm 2.42$ years. All patients were male. Patients were examined and followed at Mansoura Ophthalmic Center, Mansoura University. Both groups showed statistically significant improvement in VA, AC activity, corneal thickness and granuloma area $(p$-value $<0.001)$, which was achieved with medical treatment only in group A. However, in group B granuloma required aspiration and did not recur after that.

Conclusion Presumed trematode-induced AC granuloma is common among children living in the rural areas of Egypt. Antiparasitic medication alone was found to be effective for small-sized granulomas. Surgical aspiration is an effective adjuvant procedure to treat large-sized ones.
\end{abstract}

\section{Introduction}

Presumed trematode-induced uveitis is a newly recognized cause of intraocular granulomatous inflammation in children usually presenting with single or multiple pearl-like white nodules in the anterior chamber (AC) with or without

Amgad El Nokrashy

Amgad.ali@mans.edu.eg

1 Mansoura Ophthalmic center, Mansoura University, Mansoura, Egypt

2 UCL/Institute of Ophthalmology, London, UK

3 Moorfields Eye Hospital, London, UK

4 Mansoura Ophthalmic Hospital, Ministry of Health, Mansoura, Egypt

5 Harvard Kennedy School, Harvard University, Cambridge, USA subconjunctival lesions or corneal opacities [1-4]. Intermediate uveitis with snowbanking and posterior uveitis can also be found [5]. Children who are accustomed to swimming in nearby ponds or rivers with their eyes open are at high risk of exposure to many waterborne trematodes [6]. Different trematodes have been identified as causing granulomatous reactions. In South India, the aetiology of AC granuloma in children was confirmed to be caused by a trematode named Procerovum varium [7, 8]. Two exotic species of molluscs, Mellanoides tuberculatus and Corbicula fluminea are present in enormous numbers in the riverbanks in Brazil and were responsible for an outbreak of ocular lesions in the form of AC granulomas, subconjunctival nodules and corneal opacities [9]. In addition, freshwater sponges Drulia uruguayensis and Drulia ctenosclera can also cause AC granulomas [10].

In Egypt, schistosomiasis, or bilharziasis, is a widespread disease caused by the flat trematodes of Schistosoma genus 
with relatively rare ocular involvement. It was reported to produce granuloma reaction around eggs or adult worms at different ocular tissues. The first case was reported in Egypt by Sobhy in 1928 in the form of bilharzial conjunctival granuloma. Other ocular lesions reported in literature were keratitis, chronic conjunctivitis, iritis, chronic uveitis, vitreous opacities, multifocal choroiditis, albuminuric retinitis, chorio-retinitis, subretinal granuloma and pre-retinal haemorrhage [11, 12]. Schistosoma mansoni was also observed and isolated from AC angle [13].

Organic enrichment of the water resulting from poor sanitation is probably the key factor that initiated a cycle of ecological imbalance that results in human infection [9]. A positive correlation was found between the geographical distribution of patients' residencies close to ponds with snail habitats and such granulomatous reactions [14].

The pathogenesis of ocular lesions can be due to direct damage from the infectious agents or indirectly by toxic products or ectopic parasitism of the pre-adult or adult stages. Various theories have been postulated about the different routes by which the trematode's ova or adult worms can reach the systemic circulation and then lodge in ectopic sites such as the eyes. The most acceptable theory is that the cercariae (infective stage) develop to maturity and lay their eggs directly in the veins of the richly vascularized conjunctiva, deep episcleral plexus, the limbal structures and/or the AC. The presence of trematode's ova in the eye can produce granuloma formation and inflammatory sequelae [11, 15]. The granuloma is composed of neutrophils, eosinophils, mononuclear cells, lymphocytes, macrophages, multinucleated giant cells and fibroblasts [16]. As the immune response quietens, collagen deposits replace the granuloma. However, a disturbance in this process may lead to excessive collagen deposits forming fibrotic lesions [17].

The diagnosis of ocular parasitosis is much more difficult when the patient is not a definite host but is instead an intermediate or accidental host. In such cases, ova identification in stool is of no use. Serum samples are also unreliable mainly because of cross-reactive antigens [18]. Molecular diagnostic tests can play a vital role in overcoming these obstacles and may lead to a precise diagnosis [19]. PCR results of testing for granuloma samples were shown to be positive for trematode DNA by using quantitative real-time PCR $[2,7]$.

Different treatment modalities have been tried for the AC granulomas, including topical corticosteroids, which were the main line of treatment. Recalcitrant cases may additionally receive systemic corticosteroid starting at a daily dose of $1 \mathrm{mg} / \mathrm{kg}$ and then gradual tapering over a period of 3-6 weeks [1]. Many cases, however, did not respond well to corticosteroids and additionally developed steroid-related complications as cataract and glaucoma [20, 21]. Limbal cryotherapy was tried in some cases, under a short general anaesthetic, and applied within one clock hour of the base of the granuloma (three applications each lasting for $15 \mathrm{~s}$ ) followed by a course of topical steroids. Following cryotherapy, anterior uveitis regressed within 6 weeks, but was associated with corneal opacity and peripheral anterior synechiae at the granuloma site [20].

The activity of praziquantel as a broad-spectrum antihelmintic for systemic infections was first discovered in 1972. Ocular lesions were claimed to have responded to antischistosomal treatment [11, 12]. A single oral dose of $40 \mathrm{mg} / \mathrm{kg}$ is useful for mass treatment of trematode-induced infections with a high cure rate [22, 23]. Surgical aspiration is another alternative for granuloma not responding to treatment due to the fact that leaving untreated or unresolved granuloma for a long duration ends by collagen deposition and fibrosis leaving behind a permanent, cosmetically unacceptable scar with potential effects on vision.

This study aimed to evaluate the efficacy of systemic antiparasitic medications alone or in combination with surgical aspiration in the management of trematode-induced anterior granulomatous uveitis.

\section{Methods}

This prospective study included patients who were diagnosed with granulomatous anterior uveitis and had a recent history of swimming in local canals and ponds. The study was carried according to the Declaration of Heliniski with approval of IRB (Reference: MD/16.08.26, new modalities in uveitis treatment). Patients were recruited from the uveitis clinic, Mansoura Ophthalmic Center, Faculty of Medicine, Mansoura University. Patients' demographic characteristics, medical and ocular history were recorded. Baseline complete ocular examination was performed and involved best corrected visual acuity (VA) using Landolt $\mathrm{C}$ chart and is presented as log minimum angle of resolution for statistical analysis, slit lamp anterior segment examination (with attention to cornea, bulbar conjunctiva and AC), intraocular pressure measurement, corneal pachymetry and fundus examination. Systemic work-up was performed to rule out other causes of granulomatous uveitis. These tests included chest X-ray, Mantoux (PPD) test, and stool and urine analysis. Being endemic in Egypt, schistosomiasis (Bilharziasis) titre using indirect haemagglutination (IHA) with a cut-off titre of 1:80 (IHA ${ }^{1: 80}$ ) was added to the previous list and was done at baseline and last visit.

Inclusion criteria included patients under 18 years of age, who were diagnosed with active granulomatous anterior uveitis attributable to presumed trematodal infection. Exclusion criteria involved those who showed a history of previous ocular surgery, trauma, fibrosed granuloma with inactive inflammation and any patient with a history of 
Table 1 Demographic and baseline characteristics

\begin{tabular}{ll}
\hline Number of patients (eyes) & $30(30)$ \\
Age, mean in years (range) & $13.4(4-16)$ \\
Gender, male patients (\%) & $30(100 \%)$ \\
Unilateral disease $(\%)$ & $30(100 \%)$ \\
Eye enroled, $n$ eyes $(\%)$ & \\
OD & $15(50 \%)$ \\
OS & $15(50 \%)$ \\
Bilharziasis titre, $n$ patients $(\%)$ & \\
Negative $(<1: 80)$ & $3(10 \%)$ \\
Positive $(>1: 80)$ & $27(90 \%)$ \\
Titre $1: 160$ & 14 Out of $27(51.9 \%)$ \\
Titre $1: 320$ & 8 Out of $27(29.6 \%)$ \\
Titre $1: 640$ & 1 Out of $27(3.7 \%)$ \\
Titre $1: 1280$ & 3 Out of $27(11.1 \%)$ \\
Titre $1: 2560$ & 1 Out of $27(3.7 \%)$ \\
\hline
\end{tabular}

granulomatous inflammation other than trematodal infection (Tuberculosis or sarcoidosis). Based on positivity of bilharziasis titre in the majority of our cohort, patients were started on systemic antiparasitic medication Praziquantel tablets at a single dose of $40 \mathrm{mg} / \mathrm{kg}$, which were given either in tablet form or crushed and mixed with water for younger children ( $<6$ years). This was supplemented by metronidazole with dose adjustment according to the patients' age [37 years (200 mg daily in 2 divided doses for 3 days), 7-10 years ( $300 \mathrm{mg}$ daily in 3 divided doses for 3 days) and children $>10$ years $(600 \mathrm{mg}$ daily in 3 divided doses for 3 days)].

All involved eyes were photographed at baseline (0) and all follow-up visits 2, 4, 6 and 12 weeks (last follow-up) with a slit lamp camera. Calculation of granuloma size was performed by multiplying its greatest height and greatest width using slit lamp scale to obtain the area occupied by granuloma for simplicity. We defined a clinical response as a reduction of $50 \%$ of granuloma area (halving its size). At first follow-up ( 2 weeks), initial evaluation was performed with special attention to the granuloma size where eyes were classified into two groups: group A (reduction in granuloma size $\geq 50 \%$ ) and group B (reduction in size $<$ $50 \%$ ). Both groups were re-evaluated at 4 weeks after treatment initiation by assessing VA, granuloma area, corneal pachymetry and AC activity (cells and flare) where the granuloma area remained nearly the same in group B. After obtaining informed written consent from patients' parents, surgical aspiration was performed for patients in group B who showed a reduction of $<50 \%$ in granuloma area.

Grading of AC cells and flare was based on the Standardized Uveitis Nomenclature working group grading scheme. Aqueous cell grading was determined using a highintensity $1 \times 1 \mathrm{~mm}$ slit beam as follows: $0=<1,0.5=1-5$ cells, $1=6-15$ cells, $2=16-25$ cells, $3=26-50$ cells, and $4=>50$ cells. Aqueous flare grading was defined as follows: $0=$ none, $1=$ faint, $2=$ moderate with clear iris and lens details, $3=$ marked with hazy iris and lens details, and $4=$ intense with fibrin deposits. Improvement was defined as a two fold decrease in the AC cells and flare or a decrease to inactive (grade 0) [24]. Both clinical grading and granuloma size were performed by two examiners to ensure consistency, and if there was any conflict a third opinion was taken.

Corneal pachymetry was assessed using the pentacam (TMS-5, Tomey GmbH) to measure the baseline corneal thickness of the diseased eye and compare it in relation to the normal eye (to detect thickness difference between both eyes). We then followed up the diseased eye at 2, 4, 6 and 12 weeks.

\section{Statistical analysis}

Data were collected in an Excel spreadsheet and analysed using SPSS statistical software version 22 (IBM, Chicago, IL). A test for normality (Shapiro-Wilk) was performed for each variable where there was abnormal distribution. Changes in the variables in each group were compared with baseline using Wilcoxon signed-ranks test. However, Mann-Whitney test was used to compare variables between group A and B at each follow-up visit. A $p$-value of $<0.05$ was considered statistically significant. Results are presented as mean $\pm \mathrm{SD}$ and median.

\section{Results}

From all patients who attended uveitis outpatient clinic at Mansoura Ophthalmic Centre between 2016 and 2017, a total of 30 eyes of 30 patients met the inclusion criteria.

\section{Demographic and baseline characteristics}

The recruited patients had an average age of $13.4 \pm 2.42$ years (range from 4 to 16 years), $100 \%$ were males with unilateral involvement and all of them resided in rural villages around Mansoura city. All subjects reported the same history of swimming in local ponds and canals around the area of their location and were presented to the clinic days to weeks after exposure. Of our patients, $90 \%$ were positive for bilharziasis antibodies (12 in group A, 15 in group B) and $10 \%$ ( 3 cases) were negative (all in group A). Stool analysis revealed Entamoeba histolytica in 12 patients (40\%), Enterobius vermicularis in 8 patients (26.7\%), Ascaris in 6 patients (20\%) and Blastocystis hominis in one patient (3\%). Demographic and baseline characteristics are summarized in Table 1. 


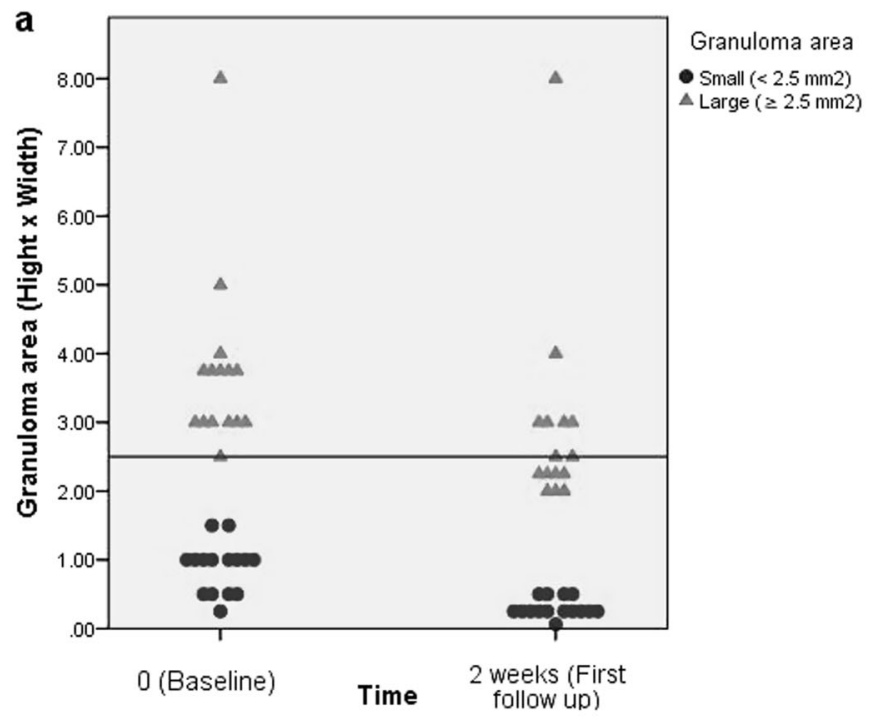

b
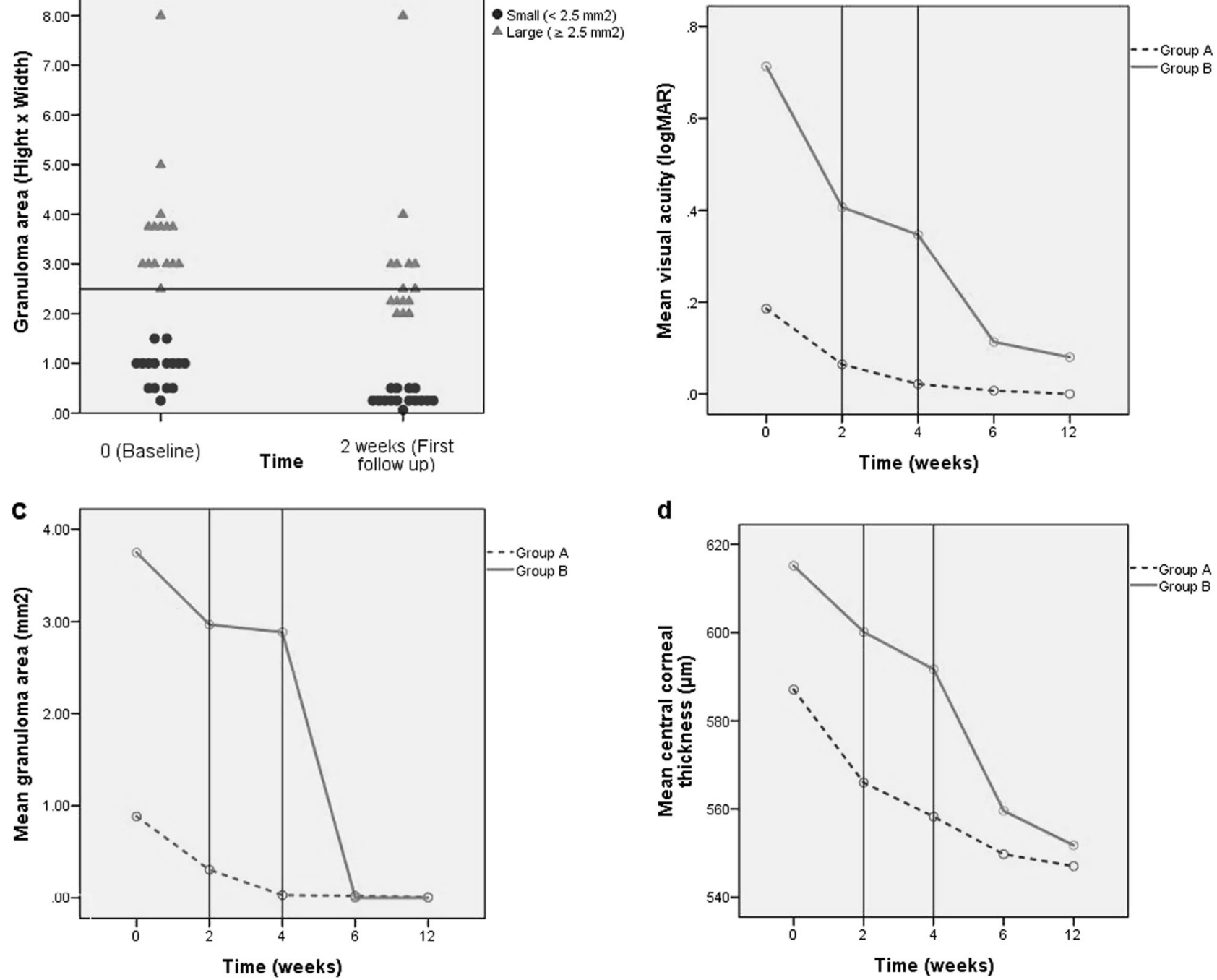

d

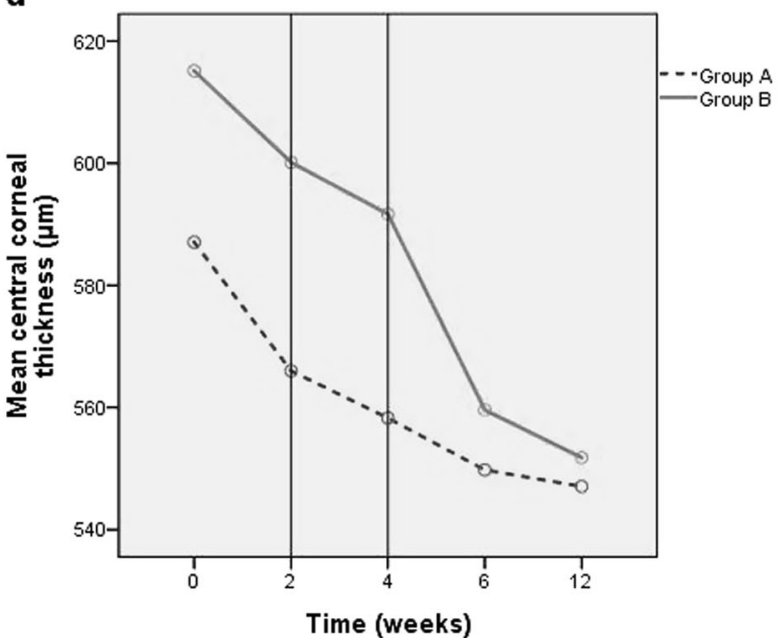

Fig. 1 Area of anterior chamber granuloma at baseline and 2 weeks after initiation of antiparasitic treatment (a). Change in mean visual acuity, mean granuloma area and mean central corneal thickness between groups A and B from baseline (0) to last visit (12 weeks) (b, c, d)

After receiving antiparasitic medical treatment (praziquantel and metronidazole), patients were reviewed after 2 weeks (first follow-up) and divided based on their clinical improvement in terms of the granuloma area into two groups: group A or small-sized granuloma patients $(<2.5$ $\mathrm{mm}^{2}$ ) who continued on antiparasitic medications only $(n=$ $15)$ and group B or large-sized granuloma patients $(\geq 2.5$ $\mathrm{mm}^{2}$ ) who then underwent surgical aspiration $(n=15)$ (Fig. 1a).

The data of the outcome parameters for both groups were collected at baseline, 2, 4, 6 and 12 weeks to be compared and analysed.

\section{Visual acuity}

Baseline mean values of VA represented as $\log$ MAR was $0.19 \pm 0.21$ for group $\mathrm{A}$, whereas it was $0.71 \pm 0.31$ for group B. The mean VA at 2 weeks was $0.06 \pm 0.09$ for group $\mathrm{A}$ and $0.41 \pm 0.23$ for group $\mathrm{B}$, and showed statistically significant improvement $(p=0.004$ and 0.001, respectively). At 4 weeks, VA continued to improve and showed statistically significant improvement compared with the baseline ( $p=0.003$ and 0.001 , respectively). At 6 weeks ( 2 weeks after surgical aspiration of granuloma in group B), the mean VA was $0.007 \pm 0.03$ and $0.11 \pm 0.2$ for group $\mathrm{A}$ and $\mathrm{B}$, respectively. VA continued to improve until 12 weeks (last follow-up) and its mean value was 0.0 and $0.08 \pm 0.2$ in group $\mathrm{A}$ and $\mathrm{B}$, respectively (Fig. 1b).

\section{AC activity}

The AC inflammatory reaction represented in grades of cells and flare was non-statistically significant higher in group B than group A at the baseline. The mean grading of cells 
for group B and A were estimated for $1.7 \pm 0.5$ and $1.43 \pm$ 0.6 , respectively $(p=0.305)$. Also the mean grading for $\mathrm{AC}$ flare in group B was $1.3 \pm 0.5$ ), whereas it was $0.93 \pm 0.3$ for group A $(p=0.148)$. Statistically significant improvement in AC activity was reported at 2 weeks in each group.

\section{Granuloma area}

The baseline average of granuloma area was $0.88 \pm 0.36 \mathrm{~mm}^{2}$ for group A and $3.75 \pm 1.33 \mathrm{~mm}^{2}$ for group B. At 2 weeks, the area of the granuloma was significantly reduced to $0.30 \pm$ $0.13 \mathrm{~mm}^{2}$ and $2.97 \pm 1.55 \mathrm{~mm}^{2}$, which represented $65.9 \%$ and $20.8 \%$ from the baseline granuloma area for group A and B, respectively. As there was no significant change in the area between week 2 and week $4\left(-0.09 \mathrm{~mm}^{2}\right)$, the remaining part of the granuloma was aspirated in group B at week 4 , whereas group A was achieving improvement after medical treatment with no need for intervention (Fig. 1c).

\section{Central corneal thickness}

The baseline mean central corneal thickness (CCT) was estimated for $587.1 \pm 26.8 \mu \mathrm{m}$ in group A [545.4 $\pm 28.9 \mu \mathrm{m}$ in the sound eye $(p=0.001)]$ compared with $615.1 \pm 30.8$ $\mu \mathrm{m}$ in group B [543.1 $\pm 13.5 \mu \mathrm{m}$ in the sound eye $(p=$ $0.000)]$. The statistically significant decrease in corneal thickness was observed in group A at 2, 4, 6, and 12 weeks $(p=0.000)$. Group B showed initial improvement until the fourth week with a sharp decrease in CCT observed following surgical aspiration (Fig. 1d).

Mean values of the four outcome variables at baseline, 2 weeks, 4 weeks, 6 weeks and 12 weeks are presented in Table 2. Also examples for group A and B cases before and after treatment are shown in Figs. 2 and 3, respectively.

\section{Discussion}

AC granuloma has a wide range of aetiologies. A newly recognized cause of granulomatous anterior uveitis is now attributed to presumed trematodal infections especialy in rural areas. The major risk for this is swimming in unsanitary local ponds or rivers and this was proved by several studies $[1-3,5,7-9,14,25]$. The high prevalence of bilharziasis in Egypt, in addition to positivity of bilharziasis titre in $90 \%$ of our selected cases, encouraged us to start a treatment plan using praziquantel as a broad-spectrum antiparasitic and supplementary metronidazole with good objective and subjective improvement.

This type of inflammatory eye disease likely affects the VA given that $41.4 \%(12 / 29)$ of our patients showed poorer than 6/12 (0.3 LogMAR) VA at baseline; of those, 91.7\% (11/12) was in group B patients. Visual impairment most commonly was related to increased $\mathrm{CCT}$ and AC cells and flare.

In our analysis all of the patients were male, for cultural reasons, which make it rare to find females swimming in the river or a local pond. All patients were below 16 years; the reason for that was attribuited to lower immunity and their high risk of infection from exposure to contaminated water than adults. The demographic features of patients treated only medically compared with those treated initially medical followed by surgical aspiration were similar in terms of age, gender and residency. Twenty-seven out of 30 patients were positive for Bilharziasis titre using IHA ${ }^{1: 80}$. IHA ${ }^{1: 80}$ was reported to have sensitivity of $92-94.7 \%$ and specificity of $94.7 \%$ [26]. The test was done at baseline and last follow-up, and a titre $>1: 80$ was considered positive. Fourteen cases had a titre of 1:160 (6 group A and 8 group B), 8 cases with a titre of 1:320 (5 group A, 3 group B) and higher titre of $>1: 640$ was observed in 5 cases (1 group $A$ and 4 group B). Negative results (titre $<1: 80$ ) were reported in all cases at last visit. However, there was no correlation between higher bilharziasis titre and disease severity, granuloma characteristics or response to treatment.

All cases showed unilateral involvement with only single nodule at the lower angle of AC (6 O'clock) but this is not the case in other studies that reported bilateral involvement in $3.5-5 \%$ of their cases $[1,4]$ or more than one nodules [1].The disease seems to have a high incidence in Egypt where it contributed to $22.2 \%$ of anterior uveitis causes in a cohort study from Egypt [4]. Because of the dry hot climate, we have noticed outbreaks of such inflammation in summer where boys find their way to the river tributaries to bathe in escaping from hot weather. Stool analysis revealed different parasites (Ascaris, E. histolytica, E. vermicularis and B. hominis) in $90 \%$ of our patients but we did not find any correlation between that and the granuloma, apart from the case with $B$. hominis parasite in stool analysis experienced the largest granuloma in our case series $\left(8 \mathrm{~mm}^{2}\right)$.

Based on granuloma size, which was represented as area for simplicity, a threshold of $2.5 \mathrm{~mm}^{2}$ granuloma area was used to categorize individuals into groups $\mathrm{A}$ and $\mathrm{B}$. The choice of this threshold was based on a reduction of $\geq 50 \%$ of granuloma area at week 2 in comparison with its baseline value.

Objectively, all patients showed improvement of outcome variables. VA improved significantly in group A in which $100 \%$ of patients gained vision better than $6 / 12$ at week 2, whereas in group B only $53.3 \%$ gained that vision at the same time. AC activity in both groups showed also improvement in both groups by the end of the second week of treatment. At week 2, the mean granuloma area was reduced by $65.9 \%$ of its original area in group A but only by $20.8 \%$ in group B. The mean CCT in group A improved 


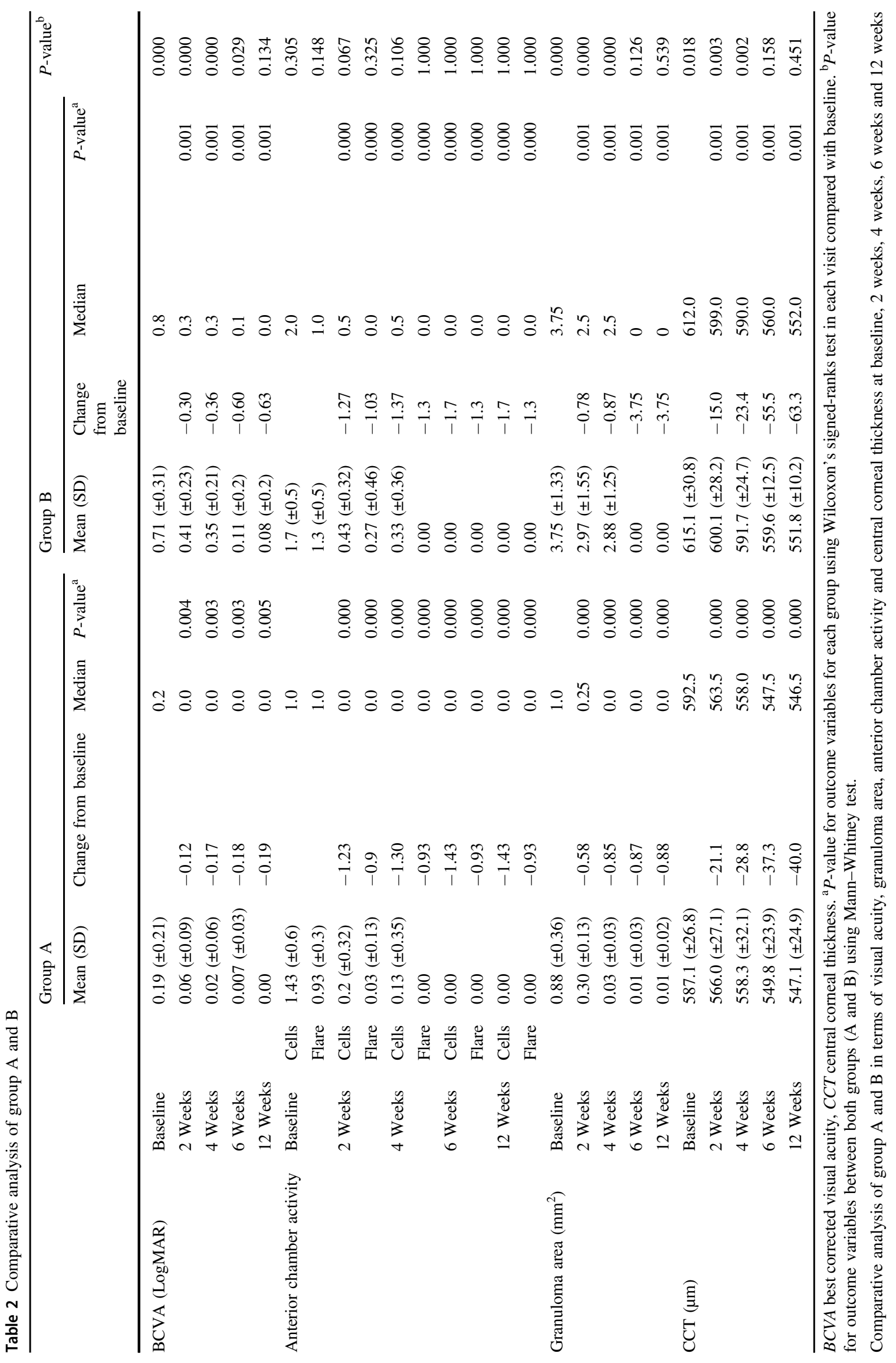



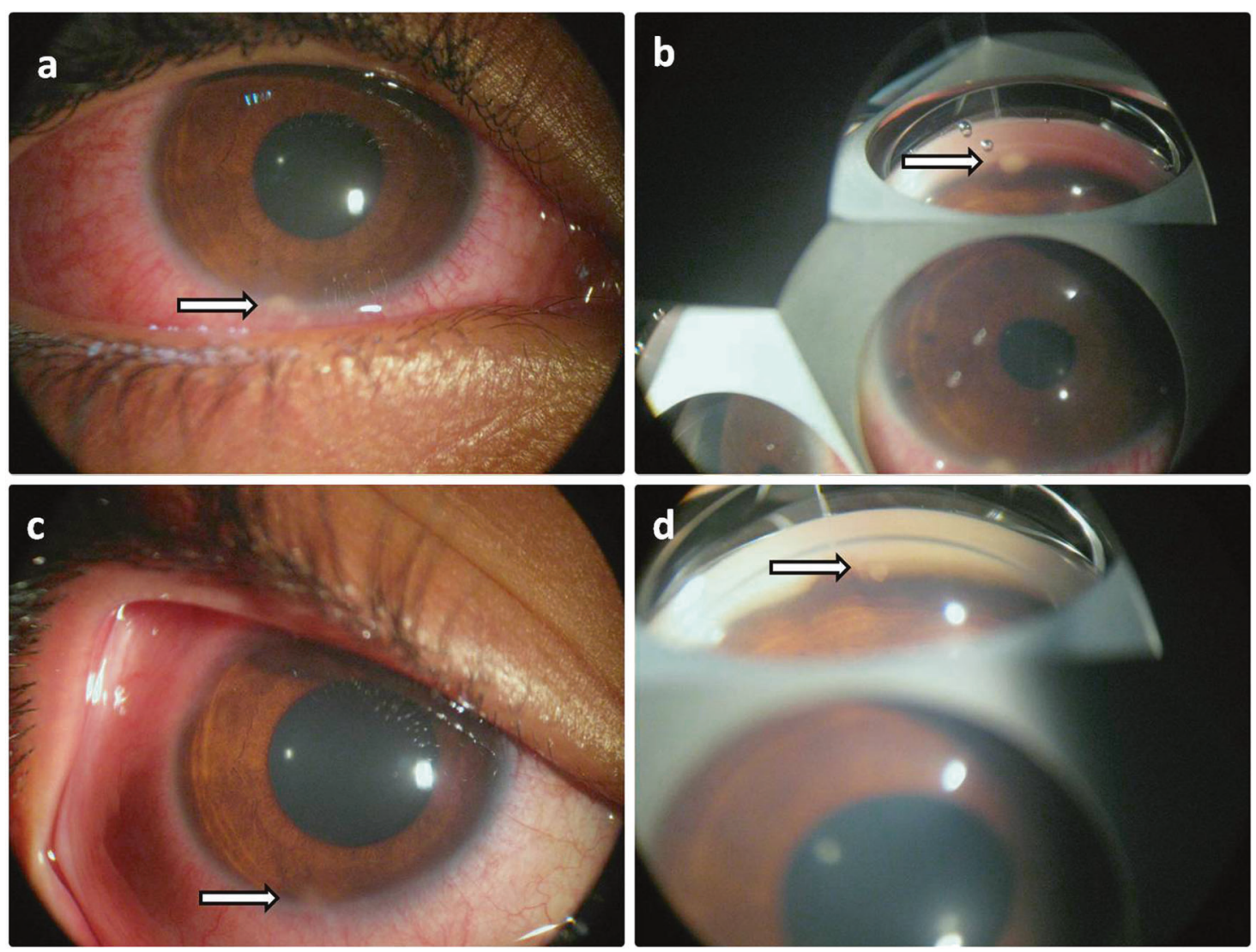

Fig. 2 A patient from group A shows small sized granuloma with slit lamp photo (a) and gonioscopy (b) before treatment and after treatment (c, d) with reduction in granuloma size and ciliary injection
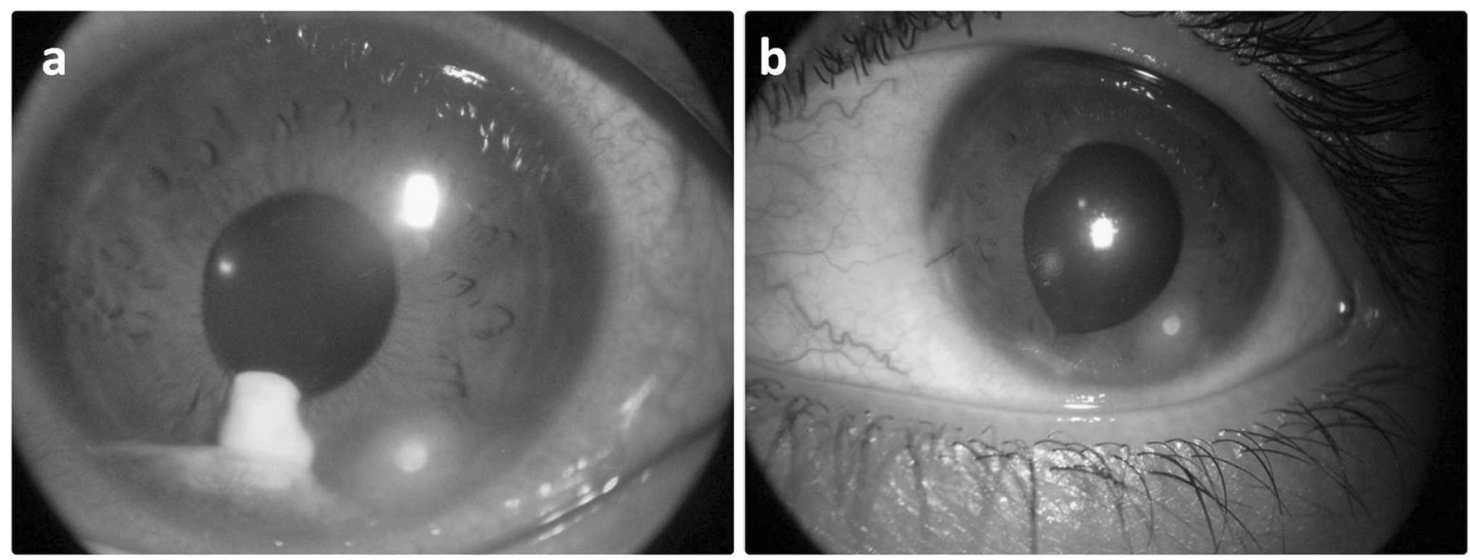

Fig. 3 A patient from group B before (a) and after (b) surgical aspiration with complete disappearance of the granuloma and ciliary injection

by $-21.1 \mu \mathrm{m}[52.75 \%$ reduction of its increased thickness $(40 \mu \mathrm{m})]$ after 2 weeks from baseline compared with -15 $\mu \mathrm{m}$ in group B [23.7\% reduction of its increased thickness $(63.3 \mu \mathrm{m})]$; there was continuous improvement in CCT in group $\mathrm{A}$, which decreased by $-28.8 \mu \mathrm{m}$ by the end of fourth week (72\%) compared with $-23.4 \mu \mathrm{m}(36.9 \%)$ in group B. These correlated parameters showed continuous improvement throughout the follow-up period (12 weeks) in group A, whereas in group B there was an early improvement that was accentuated by doing surgical aspiration at week 4. In a study by Ozdamar et al. [27], they have demonstrated that patients with active uveitis have increased CCT when compared with control group and inactive group. Also, Heinz et al. [28] found that CCT 
increases during active phases of anterior uveitis and it returned to normal after the inflammation reduced.

The use of antiparastic medications has been reported in treatment of different types of parasitic-induced uveitis. A case of bilateral uveitis secondary to intraocular filariasis was treated by diethylcarbamazine and albendazole in addition to corticosteroids [29]. The efficacy of albendazole in combination with systemic steroids was also reported in the treatment of five patients (seven eyes) with ocular toxocariasis with improvement of VA in all patients without uveitis flare up during the follow-up period [30].

Praziquantel is a broad-spectrum antiparasitic medication used to treat several types of systemic parasitic worm infections and was approved for medical use in the United States in 1982 [22]. In our study, we found that its use in combination with metronidazole is efficacious in treatment of AC granuloma induced by trematodal infection with reduction in the granuloma area that was obvious if it was $<2.5 \mathrm{~mm}^{2}$.

Surgical aspiration is an effective adjuvant procedure to treat large sized granuloma $\left(\geq 2.5 \mathrm{~mm}^{2}\right)$ with immediate dissolution and rapid recovery. Being an invasive procedure, surgical aspiration may be associated with some crucial side effects as hyphema, iatrogenic cataract and increased susceptibility to infections; therefore, it is worthy not to do it routinely for any granuloma but instead should be restricted to large unresolved one.

To our knowledge, this is the first study to assess the efficacy of antiparasitic medication alone or reinforced with surgical aspiration in treatment of the trematode-induced anterior uveitis in children. Also the prospective, welldesigned aspect of the study is an important positive point that adds to the strength of our study.

Our study has some limitations; first, the sample size enroled in the study was relatively small. Also the inability to perform PCR for samples obtained during surgical aspiration in group B and this was attributed to patients' inability or unwillingness to undergo additional financial overload. We already analysed the samples taken from the first five patients in group B pathologically but the results were nonspecific granulomatous reaction consisting lymphocyte, esinophil and plasma cells. Finally, our results regarding the use of Praziquantel and metronidazole should not be generalized to all patients with AC granuloma expected to be related to other specific types of trematode based on the geographical distribution and one should consider specific treatment according to the trematode prevalence in each area.

In conclusion, there is a good response of presumed trematode-induced uveitis to antiparastic medications. The choice of these medications depends mainly on the patients' residency and the suspected parasites. We use praziquantel due to the prevalence of bilharziasis in Egypt but further study of these agents for such challenging inflammatory conditions is warranted.

\section{Summary}

\section{What was known before}

- Corticosteroids were the main line of treatment for childhood granulomatous anterior uveitis either topical or systemic in recalcitrant cases, but many cases did not respond well to corticosteroids with the increased risk of steroid-related complications such as cataract and glaucoma. Limbal cryotherapy was another treatment option but was associated with corneal opacity and peripheral anterior synechiae at the granuloma site.

\section{What this study adds}

- The effective role of antiparasitic medications in treatment of anterior chamber granuloma induced by trematodal infection with reduction in the granuloma area was obvious if it was $<2.5 \mathrm{~mm}^{2}$. Surgical aspiration is an effective adjuvant procedure to treat large-sized granuloma $\left(2.5 \mathrm{~mm}^{2}\right.$ or more) with immediate dissolution and rapid recovery.

Acknowledgements We thank and appreciate the mission sector in Egypt, British council and Egyptian culture bureau in London.

\section{Compliance with ethical standards}

Conflict of interest The authors declare that they have no conflict of interest.

Publisher's note: Springer Nature remains neutral with regard to jurisdictional claims in published maps and institutional affiliations.

\section{References}

1. Rathinam SR, Usha KR, Rao NA. Presumed trematode-induced granulomatous anterior uveitis: a newly recognized cause of intraocular inflammation in children from south India. Am J Ophthalmol. 2002;133:773-9.

2. Rathinam S, Fritsche TR, Srinivasan M, Vijayalakshmi P, Read RW, Gautom R, et al. An outbreak of trematode-induced granulomas of the conjunctiva. Ophthalmology. 2001;108: 1223-9.

3. Amin RM, Goweida MB, El Goweini HF, Bedda AM, Lotfy WM, Gaballah AH, et al. Trematodal granulomatous uveitis in paediatric Egyptian patients: a case series. Br J Ophthalmol. 2017;101: 999-1002.

4. Amin RM, Goweida M, Bedda A, Kamel A, Radwan A. Clinical patterns and causes of intraocular inflammation in a uveitis patient cohort from Egypt. Ocul Immunol Inflamm. 2016;26:1-9. 
5. Volkmer-Ribeiro C, Lenzi HL, Orefice F, Pelajo-Machado M, de Alencar LM, Fonseca CF, et al. Freshwater sponge spicules: a new agent of ocular pathology. Mem Inst Oswaldo Cruz. 2006;101:899-903.

6. Johansen MV, Sithithaworn P, Bergquist R, Utzinger J. Towards improved diagnosis of zoonotic trematode infections in Southeast Asia. Adv Parasitol. 2010;73:171-95.

7. Rathinam SR, Arya LK, Usha KR, Prajna L, Tandon V. Novel etiological agent: molecular evidence for trematode-induced anterior uveitis in children. Arch Ophthalmol (Chicago, IL: 1960). 2012;130:1481-4.

8. Arya LK, Rathinam SR, Lalitha P, Kim UR, Ghatani S, Tandon V. Trematode fluke Procerovum varium as cause of ocular inflammation in children, South India. Emerg Infect Dis. 2016;22:192-200.

9. Cruz AA, Alencar VM, Medina NH, Volkmer-Ribeiro C, Gattas VL, Luna E. Dangerous waters: outbreak of eye lesions caused by fresh water sponge spicules. Eye. 2013;27:398-402.

10. Vasconcelos-Santos DV, Orefice F, Fonseca CF, Alencar LM, Almeida PJ, Lenzi HL, et al. Epidemic of unilateral panuveitis in children from Brazilian Amazonia: clinical and etiological aspects in seven patients. Int Ophthalmol. 2010;30:113-25.

11. Abboud IA, Hanna LS, Ragab HA. Experimental ocular schistosomiasis. Br J Ophthalmol. 1971;55:106-15.

12. Nimir AR, Saliem A, Ibrahim IA. Ophthalmic parasitosis: a review article. Interdiscip Perspect Infect Dis. 2012;2012:587402.

13. Newton JC, Kanchanaranya C, Previte LR Jr. Intraocular Schistosoma mansoni. Am J Ophthalmol. 1968;65:774-8.

14. Jayakumar D, Kavitha S, Rathinam S, Vasanthi G. Geomapping of trematode-induced granulomatous anterior uveitis - a newly identified cause of blindness among children in the Pudukkottai district of the Tamil Nadu State, India. Geospatial Health. 2009;4:55-63.

15. Nadjm B, Lynn WA, Lightman S. The wandering eye: eye infection in the returning traveller. Travel Med Infect Dis. 2005;3:97-103.

16. Gryseels B. Schistosomiasis. Infect Dis Clin North Am. 2012;26:383-97.

17. Hams E, Aviello G, Fallon PG. The schistosoma granuloma: friend or foe? Front Immunol. 2013;4:89.

18. Waikagul J, Dekumyoy P, Yoonuan T, Praevanit R. Conjunctiva philophthalmosis: a case report in Thailand. Am J Trop Med Hyg. 2006;74:848-9.
19. Dalimi A, Jabarvand M. Fasciola hepatica in the human eye. Trans R Soc Trop Med Hyg. 2005;99:798-800.

20. Venugopal N, Kummararaj G, Kummararaj S. Cryotherapy for presumed parasitic angle granuloma causing acute anterior granulomatous uveitis. Indian J Ophthalmol. 2013;61:135-6.

21. Butcher JM, Austin M, McGalliard J, Bourke RD. Bilateral cataracts and glaucoma induced by long term use of steroid eye drops. BMJ (Clin Res Ed). 1994;309:43.

22. Andrews P, Thomas H, Pohlke R, Seubert J. Praziquantel. Med Res Rev. 1983;3:147-200.

23. Coulibaly JT, N'Gbesso YK, Knopp S, Keiser J, N'Goran EK, Utzinger J. Efficacy and safety of praziquantel in preschool-aged children in an area co-endemic for Schistosoma mansoni and S. haematobium. PLoS Negl Trop Dis. 2012;6:e1917.

24. Jabs DA, Nussenblatt RB, Rosenbaum JT. Standardization of uveitis nomenclature for reporting clinical data. Results of the First International Workshop. Am J Ophthalmol. 2005; 140:509-16.

25. Rahimi M, Oustad M, Ashrafi A. Demographic and clinical features of pediatric uveitis at a tertiary referral center in Iran. Middle East Afr J Ophthalmol. 2016;23:237-40.

26. Van Gool T, Vetter H, Vervoort T, Doenhoff MJ, Wetsteyn J, Overbosch D. Serodiagnosis of imported schistosomiasis by a combination of a commercial indirect hemagglutination test with Schistosoma mansoni adult worm antigens and an enzyme-linked immunosorbent assay with S. mansoni egg antigens. J Clin Microbiol. 2002;40:3432-7.

27. Ozdamar Y, Berker N, Ertugrul G, Gurlevik U, Karakaya J, Ozkan SS. Is there a change of corneal thickness in uveitis with Behcet disease? Cornea. 2010;29:1265-7.

28. Heinz C, Taneri S, Roesel M, Heiligenhaus A. Influence of corneal thickness changes during active uveitis on Goldmann applanation and dynamic contour tonometry. Ophthalmic Res. 2012;48:38-42.

29. Pradhan ZS, Jacob P, Dikshit S. Management of bilateral uveitis secondary to intraocular filariasis. Arc Ophthalmol (Chicago, IL: 1960). 2011;129:1378-9.

30. Barisani-Asenbauer T, Maca SM, Hauff W, Kaminski SL, Domanovits H, Theyer I, et al. Treatment of ocular toxocariasis with albendazole. J Ocul Pharmacol Ther. 2001;17:287-94. 